Check for updates

Cite this: Chem. Sci., 2019, 10, 2186

๑ All publication charges for this article have been paid for by the Royal Society of Chemistry

Received 7th November 2018

Accepted 13th December 2018

DOI: $10.1039 / c 8 s c 04969 f$

rsc.li/chemical-science

\section{Efficient solar cells sensitized by a promising new type of porphyrin: dye-aggregation suppressed by double strapping $\dagger$}

\author{
Kaiwen Zeng, ${ }^{a}$ Yunyue Lu, $^{a}$ Weiqiang Tang, (DD ${ }^{\mathrm{b}}$ Shuangliang Zhao, (D) ${ }^{\mathrm{b}}$ \\ Qingyun Liu, (D) Weihong Zhu, (D) ${ }^{\text {a }} \mathrm{He}$ Tian ${ }^{\mathrm{a}}$ and Yongshu Xie (D) *a
}

Porphyrin sensitizers play essential roles in the development of efficient dye-sensitized solar cells (DSSCs). To further improve power conversion efficiency (PCE), it is vital to reduce undesirable dye aggregation that causes serious charge recombination and lowered open-circuit voltages $\left(V_{\text {oc }}\right)$. To this end, we herein report a new class of porphyrin-based dyes XW40 and XW41, with the porphyrin cores strapped with two circle chains. Compared with the reference sensitizer XW10 which contains a porphyrin core wrapped in four dodecoxyl chains, the double strapping in XW40 not only effectively suppresses the dye aggregation but also improves the dye loading amount. As a result, the $V_{\text {oc }}$ and photocurrent $\left(J_{\mathrm{sc}}\right)$ were improved by 19 $\mathrm{mV}$ and $0.8 \mathrm{~mA} \mathrm{~cm}^{-2}$, respectively, compared with the corresponding values of XW10, and the efficiency was improved from $8.6 \%$ obtained for XW10 to 9.3\% for XW40. To further extend the spectral response, an electron-withdrawing benzothiadiazole (BTD) unit was introduced as an auxiliary acceptor in XW41. Impressively, the onset wavelength of its IPCE spectrum was dramatically red-shifted to $830 \mathrm{~nm}$. However, the extended $\pi$-conjugation framework results in aggravated dye aggregation, and thus a lowered efficiency of $8.2 \%$ was obtained for XW41. Through a combined approach of coadsorption and cosensitization, the efficiencies were dramatically enhanced to $10.6 \%$ and $10.2 \%$ for XW40 and XW41, respectively, as a result of simultaneously enhanced $V_{\mathrm{oc}}$ and $J_{\mathrm{sc}}$. The results of this work provide a novel strategy for developing efficient DSSCs by employing strapped porphyrin dyes.

\section{Introduction}

Because of the increasingly serious energy shortage problems associated with the exhaustion of fuel energy resources, inexhaustible clean energy sources are highly desired. In this respect, solar energy has been considered as the most viable option for sustainable development of human society. Among the photovoltaic devices, dye-sensitized solar cells have drawn considerable attention over the past two decades, showing advantages of relatively low-cost, eco-friendliness, and relatively high PCE. ${ }^{1}$ Since Grätzel and coworkers first reported a ruthenium dye based DSSC with a PCE of $7.1 \%$ in $1991,{ }^{2}$ numerous efforts have been devoted to improve cell efficiency. For this purpose, porphyrin and their derivatives have been developed

${ }^{a}$ Key Laboratory for Advanced Materials and Feringa Nobel Prize Scientist Joint Research Center, School of Chemistry and Molecular Engineering, East China University of Science \&Technology, 130 Meilong, Shanghai 200237, China. E-mail: yshxie@ecust.edu.cn

${ }^{b}$ School of Chemical Engineering and State Key Laboratory of Chemical Engineering, East China University of Science and Technology, Shanghai, 200237, China

${ }^{c}$ College of Chemical and Environmental Engineering, Shandong University of Science and Technology, Qingdao, P. R. China

$\dagger$ Electronic supplementary information (ESI) available. See DOI: $10.1039 / \mathrm{c} 8 \mathrm{sc} 04969 f$ as promising sensitizers because of their facile structural modification and intrinsic strong absorption capability, showing an intense Soret band and moderate $\mathrm{Q}$ bands in the ranges of 400-450 $\mathrm{nm}$ and 550-650 nm, respectively. ${ }^{3-5}$

Highly efficient porphyrin sensitizers usually feature a donor- $\pi$-acceptor (D- $\pi-A)$ configuration. ${ }^{6}$ Such structures exhibit excellent intramolecular charge transfer (ICT) properties, and tunable optical, physical and electrochemical properties. $^{7}$ However, simple porphyrin dyes usually exhibit poor absorption in the near infrared (NIR) region. To enhance lightharvesting ability in the NIR region, porphyrin sensitizers with extended conjugation frameworks have been developed..$^{\mathbf{8 - 1 0}}$ However, this approach usually aggravates the undesired dye aggregation, which may result in serious charge recombination, low electron-injection efficiency, and poor cell performance. ${ }^{\mathbf{1 1 - 1 5}}$ To overcome such a problem, efficient porphyrin dyes have been developed by wrapping the porphyrin macrocycle with four $o$-alkoxyl moieties on the meso-phenyl substituents. ${ }^{\mathbf{1 6 - 2 0}} \mathrm{In}$ this respect, we have designed XW10 using a phenothiazinebased donor, and an efficiency of $8.6 \%$ was achieved for the individual dye. ${ }^{33}$

On the basis of XW10, with the purpose of further suppressing the dye aggregation effect, we herein report a new class of porphyrin sensitizers XW40 and XW41 (Fig. 1) with double 

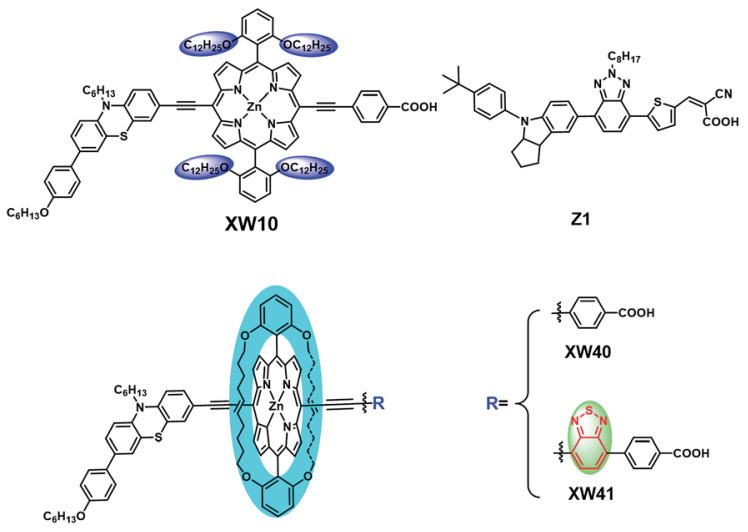

Fig. 1 Molecular structures of the porphyrin dyes and the cosensitizer Z1.

straps. Compared with XW10, the straps in XW40 are favourable for suppressing dye aggregation, resulting in simultaneously improved $V_{\mathrm{oc}}$ and $J_{\mathrm{sc}}$ and an efficiency of 9.3\%, obviously higher than the $8.6 \%$ obtained for XW10. On this basis, XW41 was synthesized by introducing an auxiliary benzothiadiazole (BTD) moiety with the purpose of further extending the absorption spectrum. ${ }^{\mathbf{9 2 1 - 2 4}}$ As a result, the onset wavelength of photocurrent response was red-shifted to $830 \mathrm{~nm}$. Upon cosensitization with an organic dye, Z1, high efficiencies of $10.6 \%$ and $10.2 \%$ were achieved for XW40 and XW41, respectively, based on the traditional iodine electrolyte. Although circle chain strapping has been demonstrated to be effective in developing organic dyes, ${ }^{25,26}$ and strapped porphyrins have been reported for fabrication of OLEDs, and to achieve charge transfer and sensitization behavior on semiconductor films, ${ }^{27-29}$ this approach has not yet been employed in designing efficient porphyrin dyes. The results of this work provide an effective novel strategy for designing efficient porphyrin dyes by introducing double straps.

\section{Results and discussion}

\section{Synthesis of dyes}

The synthesis routes are depicted in Scheme 1 and Scheme S1. $\dagger$ 2,6-bis(Hex-5-en-1-yloxy)benzaldehyde 1 was prepared from commercially available 2,6-dihydroxybenzaldehyde in $92 \%$ yield. Acid-catalysed condensation of 1 with dipyrromethane followed by DDQ oxidation provided 5,15-bis(2,6-bis(hex-5-en-1yloxy)phenyl)porphyrin 2. Bromination of 2 with NBS followed by $\mathrm{Zn}^{\mathrm{II}}$-ion insertion gave porphyrin 4 , which was subjected to ring-closing metathesis (RCM) using the second-generation Grubbs catalyst, affording the key intermediate doubly strapped porphyrin 5. Then, it was successively reacted with the ethynyl derivatives of phenothiazine, and the acid ester of the acceptor through Sonogashira reactions to introduce the donor and the acid ester of the acceptor, respectively, in the meso-positions of the strapped porphyrin framework. Finally, the target dyes were obtained through hydrolysis of the corresponding esters. All the compounds were systematically characterized by ${ }^{1} \mathrm{H}$ NMR, ${ }^{13} \mathrm{C}$ $\mathrm{NMR}$, and mass spectrometry (HRMS; ESI $\dagger$ ). In addition, the key
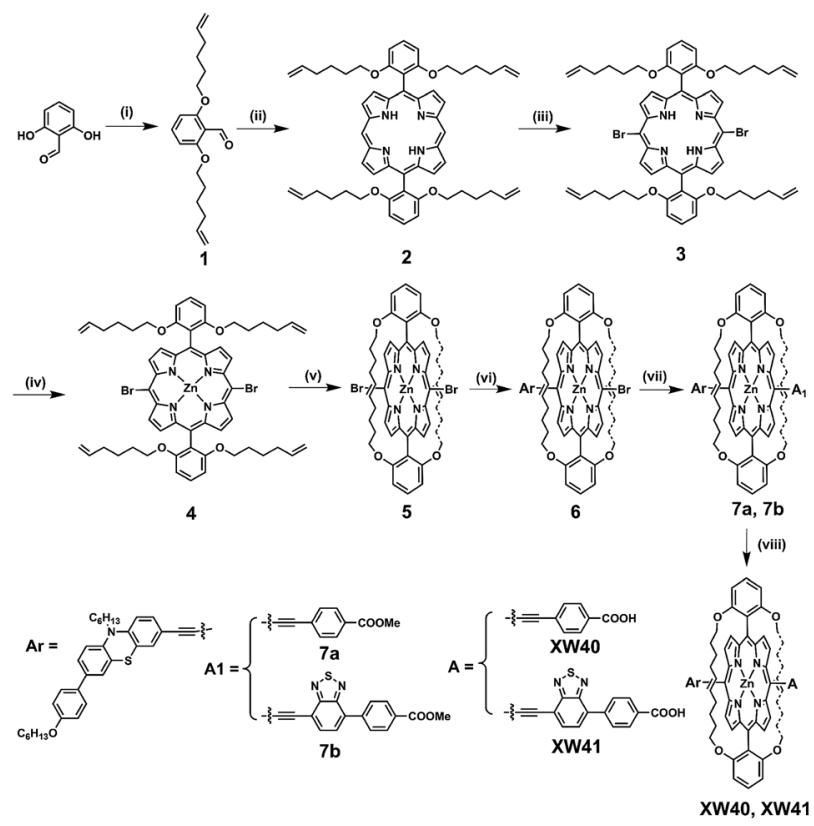

Scheme 1 Synthetic routes to sensitizers XW40 and XW41. Reaction conditions: (i) 6-bromo-1-hexene, $\mathrm{K}_{2} \mathrm{CO}_{3}, \mathrm{DMF}$; (ii) TFA, DDQ, $\mathrm{Et}_{3} \mathrm{~N}$, $\mathrm{CH}_{2} \mathrm{Cl}_{2}$; (iii) NBS, $\mathrm{CH}_{2} \mathrm{Cl}_{2}$; (iv) $\mathrm{Zn}(\mathrm{OAC})_{2} \cdot 2 \mathrm{H}_{2} \mathrm{O}, \mathrm{CH}_{2} \mathrm{Cl}_{2}, \mathrm{CH}_{3} \mathrm{OH}$; (v) 2nd generation Grubbs catalyst, $\mathrm{CH}_{2} \mathrm{Cl}_{2}$; (vi) 3-ethynyl-10-hexyl-7-(4(hexyloxy)phenyl)-10H-phenothiazine, $\mathrm{AsPh}_{3}, \mathrm{Pd}_{2}\left(\mathrm{dba}_{3}, \mathrm{THF} \mathrm{Et}_{3} \mathrm{~N}\right.$; (vii) for 7a: methyl-4-ethynylbenzoate, $\mathrm{Pd}_{2}\left(\mathrm{dba}_{3}, \mathrm{AsPh}_{3}, \mathrm{Et}_{3} \mathrm{~N}\right.$, THF; for 7b: methyl 4-(7-ethynylbenzo[c][1,2,5]thiadiazol-4-yl)benzoate, $\mathrm{Pd}_{2}(\mathrm{dba})_{3}, \mathrm{AsPh}_{3}, \mathrm{Et}_{3} \mathrm{~N}, \mathrm{THF}$; and (viii) $\mathrm{LiOH} \cdot \mathrm{H}_{2} \mathrm{O}, \mathrm{THF}, \mathrm{H}_{2} \mathrm{O}$.

intermediate 5 was further characterized with single crystal Xray diffraction analyses (Fig. $\mathrm{S} 4 \dagger$ ).

\section{Spectral properties}

The electronic absorption spectra of the dyes in THF are shown in Fig. 2, and the corresponding absorption together with emission data are listed in Table 1.

As expected, all the porphyrin dyes exhibit a typical intense Soret band around $460 \mathrm{~nm}$ and less intense $\mathrm{Q}$ bands in the wavelength range of 600-750 $\mathrm{nm}$. Compared with XW10, doubly

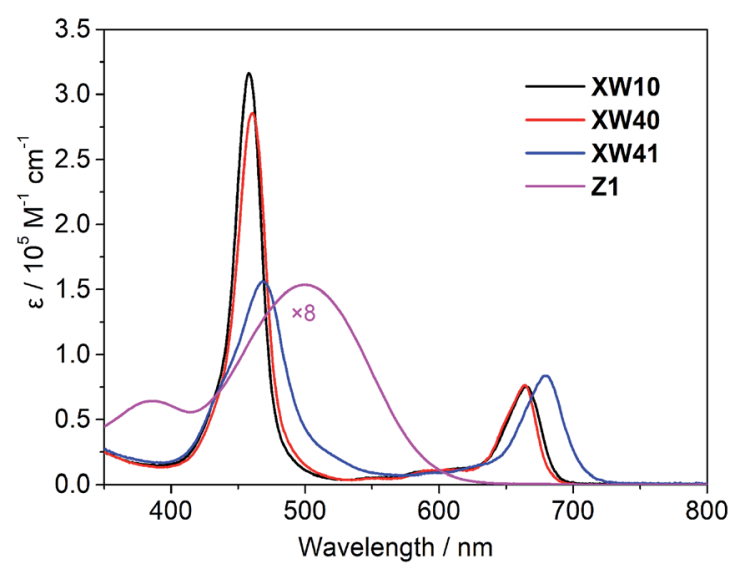

Fig. 2 Absorption spectra of the porphyrin dyes and the cosensitizer Z1 in THF. 
Table 1 Absorption spectral and electrochemical data for XW40 XW41 and Z1

\begin{tabular}{lll}
\hline Dye & $\lambda_{\max } / \mathrm{nm}\left(\varepsilon / 10^{5} \mathrm{M}^{-1} \mathrm{~cm}^{-1}\right)^{a}$ & $\lambda_{\max } / \mathrm{nm}^{b}$ \\
\hline XW10 $^{33}$ & $459(316), 591(10.4), 666(75.2)$ & 676 \\
XW40 & $461(286), 594(10.9), 664(76.6)$ & 672 \\
XW41 & $468(156), 627(14.3), 680(83.7)$ & 700 \\
Z1 & $386(8.03), 501(19.2)$ & 672
\end{tabular}

${ }^{a}$ Absorption peaks in THF $\left(4 \times 10^{-6} \mathrm{M}\right) .{ }^{b}$ Emission peaks in THF $(4 \times$ $\left.10^{-6} \mathrm{M}\right)$.

strapped porphyrin dye XW40 exhibits slightly red-shifted Soret band, and its Q bands are almost identical to those of XW10. As expected, the introduction of an auxiliary benzothiadiazole (BTD) group in XW41 dramatically improves the light-harvesting ability up to $730 \mathrm{~nm}$.

The absorption spectra of XW40 and XW41 on $3 \mu \mathrm{m}$ thick $\mathrm{TiO}_{2}$ films are shown in Fig. S1. $\uparrow$ Compared with the corresponding absorption spectra in THF, all the spectra acquired on the films show broadened absorption, beneficial for enhancing the light-harvesting ability, and the $\mathrm{Q}$ peaks are hypsochromically shifted, which can be ascribed to the deprotonation of the carboxylic acid moiety. ${ }^{30-32}$ Notably, the weak blue shoulder of the Soret band observed for XW10 was not discernible in XW40, indicating that the H-type aggregation of porphyrins on $\mathrm{TiO}_{2}$ might be insignificant. However, the re-appearance of the obvious blue-shoulder of the Soret band in the $\mathbf{x W 4 1} / \mathrm{TiO}_{2}$ spectrum indicates severe aggregation due to the introduction of the BTD group, ${ }^{\mathbf{1 6 , 3 0}}$ which is detrimental to DSSC photovoltaic performance.

\section{Electrochemical studies}

Cyclic voltammetry (Fig. S3†) was carried out to investigate the thermodynamic driving force of electron injection from the photoexcited dyes to the conduction band of $\mathrm{TiO}_{2}$, and dye regeneration by the redox electrolyte. The obtained HOMO levels corresponding to the ground state oxidation potentials of the two dyes are 0.71 and $0.72 \mathrm{~V}$ ( $v s$. NHE), respectively (Table 2). Their HOMO levels are well below the redox potential of the iodide/triiodide couple ( $\sim 0.4 \mathrm{~V} v s$. NHE), indicating thermodynamically feasible regeneration processes for the sensitizers, and the respective LUMO levels of $-\mathbf{1 . 1 5}$ and $-1.08 \mathrm{~V}$ for XW40

Table 2 Electrochemical data and the energy levels of the molecular orbitals for the dyes adsorbed on $\mathrm{TiO}_{2}$ films

\begin{tabular}{llll}
\hline Dyes & $\mathrm{HOMO}^{a} / \mathrm{V}(\mathrm{NHE})$ & $E_{0-0}{ }^{b} / \mathrm{V}$ & $\mathrm{LUMO}^{c} / \mathrm{V}(\mathrm{NHE})$ \\
\hline XW40 & 0.71 & 1.86 & -1.15 \\
XW41 & 0.72 & 1.80 & -1.08
\end{tabular}

${ }^{a}$ HOMO levels were obtained from the first oxidation potential measured in acetonitrile using $0.1 \mathrm{M}$ tetrabutylammonium hexafluorophosphate $\left(\mathrm{TBAPF}_{6}\right)$ as the electrolyte (working electrode: $\mathrm{FTO} / \mathrm{TiO}_{2}$ /dye; reference electrode: SCE; calibrated with ferrocene/ ferrocenium $\left(\mathrm{Fc} / \mathrm{Fc}^{+}\right)$as an external reference. Counter electrode: $\left.\mathrm{Pt}\right)$. ${ }^{b}$ Estimated from the wavelength at the intersection $\left(\lambda_{\text {inter }}\right)$ of normalized and emission spectra with the equation $E_{0-0}=1240 / \lambda_{\text {inter }}$. ${ }^{c}$ The LUMO was calculated from the equation LUMO $=$ HOMO $-E_{0-0}$.

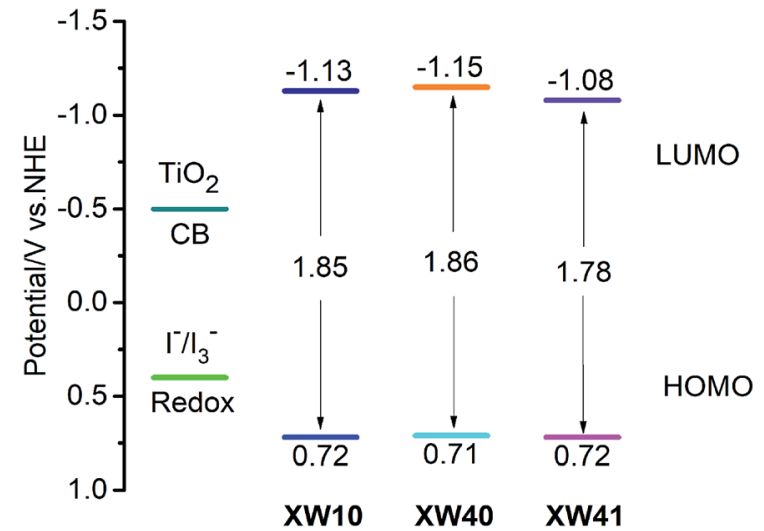

Fig. 3 Schematic energy-level diagram of XW10, ${ }^{33}$ XW40 and XW41.

and XW41 lie well above the conduction band edge of $\mathrm{TiO}_{2}$ $(-0.5 \mathrm{~V}$ vs. NHE), indicating sufficient driving forces for the electron injection processes (Fig. 3).

\section{Theoretical calculations}

To gain insight into the electron distribution of distinct porphyrin dyes, density functional theory (DFT) calculations were performed using the Gaussian 09 program package. As presented in Fig. 4, the HOMO orbitals of porphyrin dyes XW40 and XW41 are distributed over the phenothiazine donors and the porphyrin frameworks. The LUMO orbitals of XW40 are predominantly delocalized over the acceptor and porphyrin moiety, while the LUMO orbitals of XW41 are mainly delocalized over the porphyrin framework, the BTD unit and the carboxyphenyl acceptor. The obvious overlap between the HOMO and LUMO orbitals facilitates the efficient intramolecular charge-transfer from the donor to the acceptor and subsequent electron-injection from the excited dyes to the $\mathrm{TiO}_{2}$ film..$^{34-36}$

\section{Photovoltaic performance}

The porphyrin dyes were evaluated as the sensitizers for fabricating DSSCs based on the traditional $\mathrm{I}^{-} / \mathrm{I}_{3}{ }^{-}$electrolyte. Fig. 5 shows the photocurrent-voltage $(J-V)$ curves of the DSSCs, and the photovoltaic parameters are listed in Tables 3 and $S 1 . \dagger$
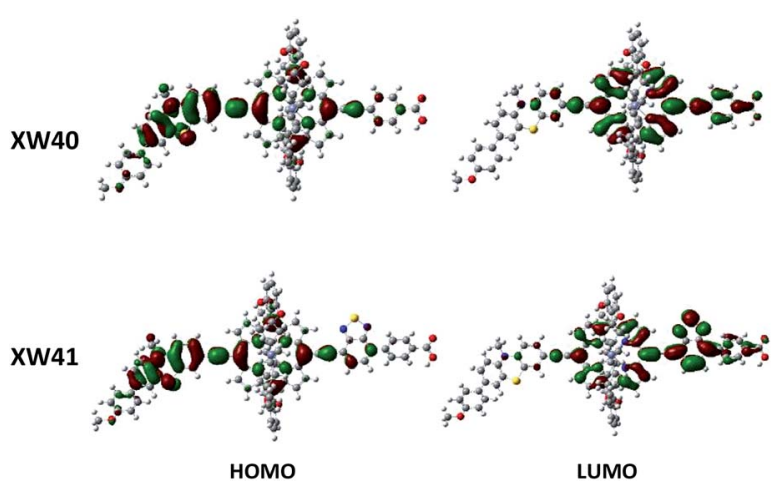

Fig. 4 Frontier molecular orbital profiles of XW40 and XW41. 


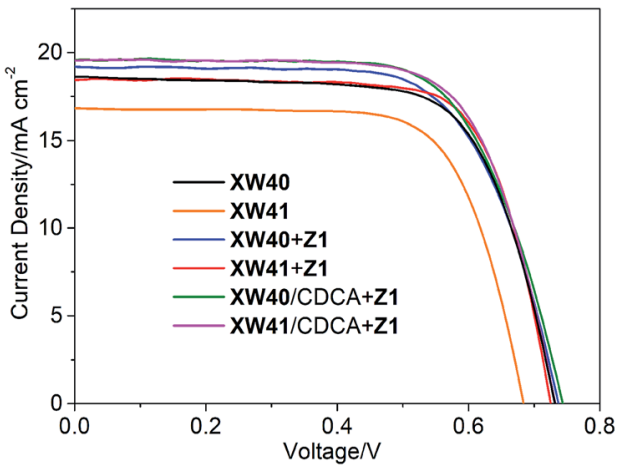

Fig. 5 J-V characteristics of the DSSCs based on Z1, XW40 and XW41.

The DSSCs based on XW40 exhibit a PCE of 9.3\%, higher than the $8.6 \%$ obtained for XW10, with $V_{\mathrm{oc}}$ and $J_{\mathrm{sc}}$ improved to $730 \mathrm{mV}$ and $18.90 \mathrm{~mA} \mathrm{~cm}{ }^{-2}$, respectively, compared to the respective values of $711 \mathrm{mV}$ and $17.90 \mathrm{~mA} \mathrm{~cm}{ }^{-2}$ obtained for XW10. ${ }^{33}$ These observations indicate that the porphyrin core strapping is favorable for suppressing the aggregation effect and reducing the charge recombination, and thus the $V_{\mathrm{oc}}$ of XW40 was significantly enhanced by $19 \mathrm{mV}$, compared to that of XW10. On the other hand, the $J_{\text {sc }}$ of XW40 was also improved despite the fact that $\mathbf{X W 4 0}$ and $\mathbf{X W 1 0}$ possess identical $\pi$-conjugation backbones and very similar absorption spectra. To further understand the enhancement of $J_{\mathrm{sc}}$, we measured the adsorption amounts of the dyes (Table 3). The loading amount of XW40 was found to be $1.99 \times 10^{-7} \mathrm{~mol} \mathrm{~cm}^{-2}, 23 \%$ higher than the $1.62 \times 10^{-7} \mathrm{~mol} \mathrm{~cm}{ }^{-2}$ measured for XW10. Hence, the higher $J_{\text {sc }}$ obtained for $\mathbf{X W 4 0}$ can be rationalized by its larger dye adsorption amount, which may be ascribed to the fact that the doubly strapped porphyrin molecule may occupy a smaller area, because the doubly strapped structure is more compact than the quadruply wrapped structure. Compared to XW40, although XW41 absorbs light over a broader range due to the presence of an extra BTD unit, it exhibits a lower PCE of 8.2\%, which may be ascribed to the lower $V_{\text {oc }}$ of $695 \mathrm{mV}$ associated

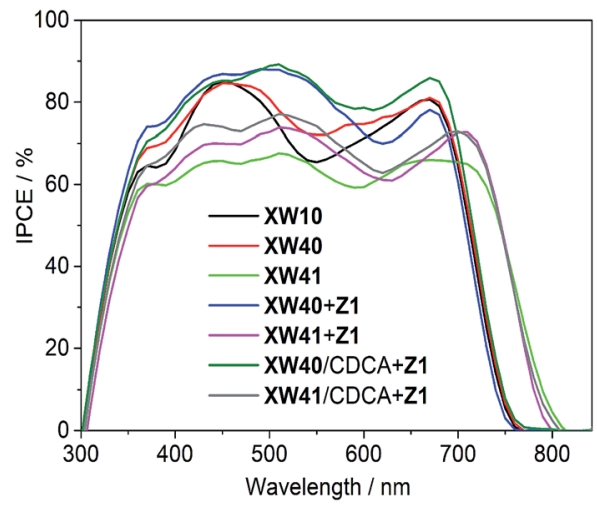

Fig. 6 IPCE action spectra for the DSSCs based on XW10, ${ }^{33} \mathrm{Z1}, \mathrm{XW} 40$ and XW41.

with the aggravated dye aggregation effect induced by the extended conjugation framework. However, the efficiency of XW41 is still higher than the $7.8 \%$ obtained for $\mathbf{X W 1 1},{ }^{33}$ in agreement with the superior anti-aggregation abilities of the doubly strapped porphyrins relative to the quadruply wrapped porphyrins.

The incident photon-to-current conversion efficiency (IPCE) spectra for the dyes were also measured to probe the efficiencies for the conversion of the absorbed sunlight to electrical current at various wavelengths. As shown in Fig. 6, the bandwidths of IPCE spectra for XW40 are almost the same as those of XW10, ${ }^{33}$ but the plateau is slightly higher than that of XW10, which is consistent with the improved $J_{\mathrm{sc}}$. Impressively, the introduction of the auxiliary BTD acceptor unit in XW41 successfully expanded the light responsive wavelengths into the NIR region with a striking onset wavelength of $830 \mathrm{~nm}$, but the plateau is obviously lowered, leading to a lowered $J_{\mathrm{sc}}$ of $16.77 \mathrm{~mA} \mathrm{~cm}^{-2}$.

It is obvious that both XW40 and XW41 exhibit obvious valleys within 500-600 $\mathrm{nm}$ in the IPCE spectra. To fill up the valleys, an organic dye, $\mathbf{Z 1}$ (Fig. 1, Table S2†), was thus synthesized and employed for co-sensitization with XW40 and XW41.

Table 3 Photovoltaic parameters of the porphyrin DSSCs under AM1.5 illumination (power, $100 \mathrm{~mW} \mathrm{~cm}^{-2}$ ). The active area is $0.12 \mathrm{~cm}^{2}$

\begin{tabular}{|c|c|c|c|c|c|c|}
\hline \multirow[b]{2}{*}{ Dye } & \multirow[b]{2}{*}{$V_{\mathrm{oc}} / \mathrm{mV}$} & \multirow[b]{2}{*}{$J_{\mathrm{sc}} / \mathrm{mA} \mathrm{cm}^{-2}$} & \multirow[b]{2}{*}{$\mathrm{FF}$} & \multirow[b]{2}{*}{ PCE (\%) } & \multicolumn{2}{|c|}{$\begin{array}{l}\text { Dye loading amount } \\
\left(\times 10^{-7} \mathrm{~mol} \mathrm{~cm}^{-2}\right)\end{array}$} \\
\hline & & & & & Porphyrin & $\mathbf{Z 1}$ \\
\hline $\mathbf{X W 1 0}^{a}$ & $711 \pm 5$ & $17.90 \pm 0.04$ & $0.684 \pm 0.005$ & $8.60 \pm 0.10$ & 1.62 & 1 \\
\hline XW40 & $730 \pm 3$ & $18.67 \pm 0.75$ & $0.683 \pm 0.021$ & $9.31 \pm 0.12$ & 1.99 & 1 \\
\hline XW41 & $695 \pm 2$ & $16.77 \pm 0.31$ & $0.701 \pm 0.006$ & $8.16 \pm 0.10$ & 2.78 & 1 \\
\hline $\mathbf{Z 1}^{b}$ & $788 \pm 3$ & $13.30 \pm 0.15$ & $0.781 \pm 0.004$ & $8.23 \pm 0.03$ & 1 & 6.47 \\
\hline $\mathbf{X W 4 0}+\mathbf{Z 1}^{c}$ & $742 \pm 1$ & $19.36 \pm 0.49$ & $0.694 \pm 0.013$ & $9.97 \pm 0.06$ & 1.62 & 3.67 \\
\hline $\mathbf{X W} 41+\mathbf{Z 1}^{c}$ & $728 \pm 3$ & $18.32 \pm 0.13$ & $0.728 \pm 0.003$ & $9.71 \pm 0.01$ & 2.65 & 1.96 \\
\hline $\mathbf{X W 4 0} / \mathrm{CDCA}+\mathbf{Z 1}^{d}$ & $748 \pm 2$ & $19.59 \pm 0.21$ & $0.719 \pm 0.004$ & $10.55 \pm 0.11$ & 1.64 & 2.73 \\
\hline $\mathbf{X W 4 1} / \mathrm{CDCA}+\mathbf{Z 1}^{d}$ & $726 \pm 2$ & $19.63 \pm 0.31$ & $0.715 \pm 0.003$ & $10.19 \pm 0.21$ & 2.39 & 3.06 \\
\hline
\end{tabular}


Upon co-sensitization, the valleys in the IPCE spectra were filled up by the contribution from the absorption of $\mathbf{Z 1}$, and thus better photovoltaic performance was achieved for both XW40 and XW41, with higher efficiencies of $10.0 \%$ and $9.7 \%$, respectively, as a result of simultaneously improved $J_{\mathrm{sc}}$ and $V_{\mathrm{oc}}$. The enhancement of $J_{\text {sc }}$ can be readily rationalized by the fact that the IPCE spectra are obviously improved upon cosensitization. On the other hand, the improved $V_{\text {oc }}$ values achieved for the cosensitization systems lie between the corresponding values obtained for the individual porphyrin dyes and the cosensitizer. These observations agree well with previous results of cosensitization systems..$^{\mathbf{3 3 , 3 8 - 4 0}}$

Generally, the utilization of CDCA as a coadsorbent is effective for suppressing dye aggregation and enhancing the photovoltaic performance. Hence, a combined strategy of coadsorption and cosensitization was employed with the purpose of further enhancing the photovoltaic performance of DSSCs. Thus, the DSSCs were fabricated by immersing $\mathrm{TiO}_{2}$ films in a cocktail solution containing the individual porphyrin dye and CDCA for $12 \mathrm{~h}$, followed by dipping in a solution containing Z1. Compared with XW40 $+\mathbf{Z 1}$ and XW41 $+\mathbf{Z 1}$, the $J_{\mathrm{sc}}$ values obtained for XW40/CDCA $+\mathbf{Z 1}$ and XW41/ CDCA + Z1 were improved to 19.59 and $19.63 \mathrm{mAcm}^{-2}$, respectively, with the efficiencies enhanced to $10.6 \%$ and $10.2 \%$, respectively. The improved $J_{\mathrm{sc}}$ values are consistent with the improved IPCE responses (Fig. 6). As we know, the presence of CDCA can suppress dye aggregation, decrease the rate of aggregation-induced non-radiative energy transfer, enhance electron injection yield, and thus improve the IPCE response. ${ }^{7}$ For $\mathbf{X W 4 0 / C D C A}+\mathbf{Z 1}$, the improved IPCE response around $500 \mathrm{~nm}$ was offset by the decreased loading amount of Z1 which features a broad absorption around $500 \mathrm{~nm}$ (Fig. S5 $†$ ). Meanwhile, the loading amount of XW40 remains almost unchanged. As a result, the IPCE response was improved mainly in the Q band region. For XW41/CDCA + Z1, the loading amounts of XW41 and $\mathbf{Z 1}$ were decreased and increased, respectively, compared with the corresponding values for the cells based on XW41 + Z1. Consistently, the IPCE response was improved mainly within the wavelength range of 400-600 nm.

\section{Electrochemical impedance spectroscopy analysis}

The $V_{\mathrm{oc}}$ for a DSSC is dependent on the $\mathrm{TiO}_{2}$ conduction band edge $\left(E_{\mathrm{CB}}\right)$ and the charge recombination process, which can be inferred from the chemical capacitance $\left(C_{\mu}\right)$ and electron lifetime $(\tau)$, respectively. To provide further insight into the improved photovoltaic behavior of the doubly strapped porphyrin dyes, electrochemical impedance spectroscopy (EIS) was conducted under dark conditions. Thereby, the $C_{\mu}$ and $\tau$ values were obtained through fitting the EIS spectra.

As shown in Fig. 7a, the devices based on XW40 showed similar $C_{\mu}$ values with those of XW10 at a given $V_{\text {oc }}$. However, the electron lifetimes of XW40 were found to be obviously longer than those of XW10 (Fig. 7b), indicating a slower interfacial charge recombination process, which is consistent with the significantly enhanced $V_{\text {oc }}$ for the doubly strapped
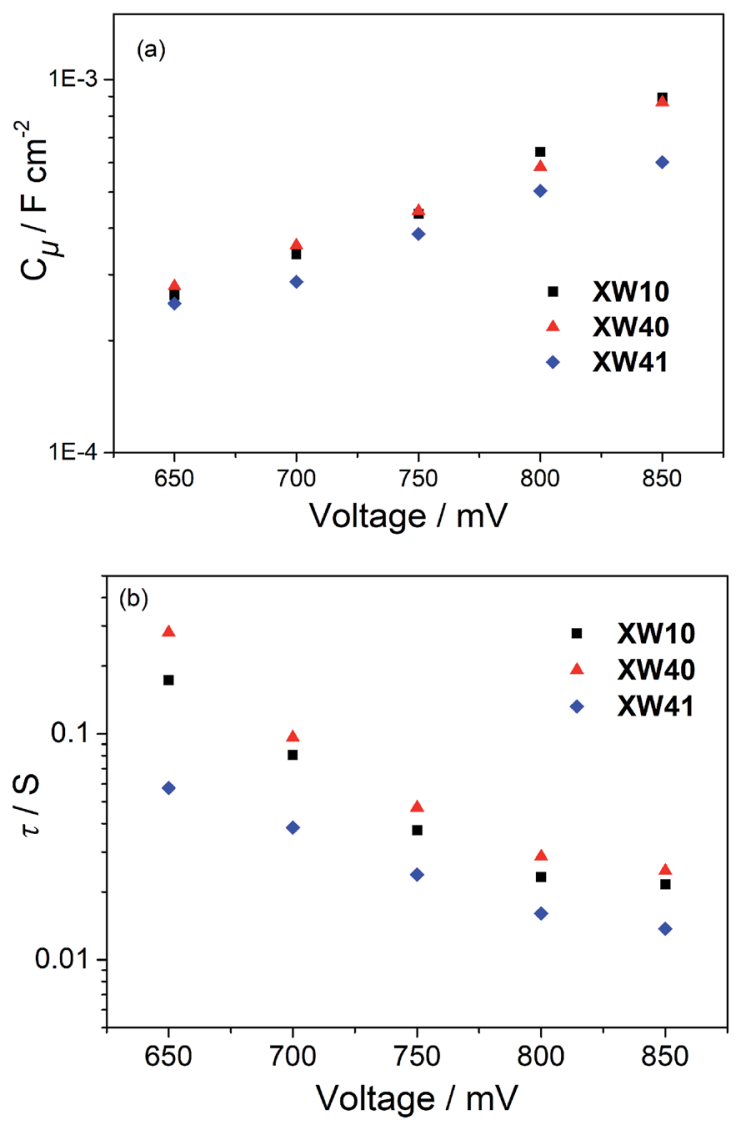

Fig. 7 Plots of (a) $C_{\mu}$ and (b) $\tau$ versus bias voltages of DSSCs based on XW10, XW40, and XW41.

porphyrin dyes. On the other hand, the $C_{\mu}$ values of XW41 are lower than those of XW40, which is favorable for improving the $V_{\text {oc }}$. However, the electron lifetimes for XW41 are much shorter than those of XW40 which is unfavorable for the $V_{\text {oc }}$. As a result of the contradictory effects, XW41 exhibits a lower $V_{\text {oc }}$ than
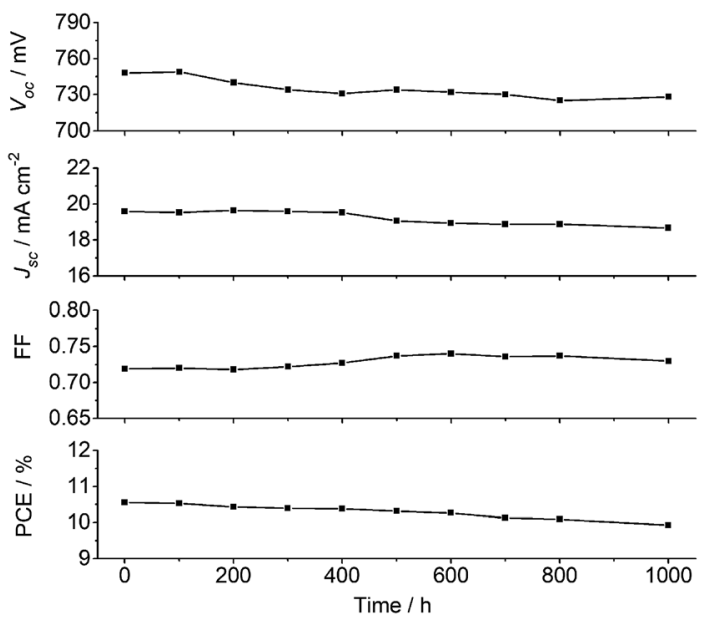

Fig. 8 Variation of the photovoltaic parameters $\left(V_{\mathrm{OC}}, J_{\mathrm{SC}}, \mathrm{FF}\right.$, and PCE) with aging time for the DSSC device based on XW40/CDCA + Z1 measured under AM 1.5 illumination $\left(100 \mathrm{~mW} \mathrm{~cm}^{-2}\right)$ at $60{ }^{\circ} \mathrm{C}$ over $1000 \mathrm{~h}$. 
XW40, indicating that the $V_{\text {oc }}$ values are predominantly governed by the electron lifetimes. These results are consistent with the more severe dye aggregation of XW41 associated with the extended conjugation framework due to the presence of the additional BDT unit.

\section{Photostability}

In addition to high efficiency, long-term stability is a critical requirement for practical use of DSSCs. Here, the $V_{\mathrm{oc}}, J_{\mathrm{sc}}, \mathrm{FF}$ and PCE values for the XW40/CDCA + Z1 cells were recorded over a period of $1000 \mathrm{~h}$ under one sun soaking at $60^{\circ} \mathrm{C}$ (Fig. 8). After $1000 \mathrm{~h}$ light soaking, more than $90 \%$ of the initial PCE value was retained, indicating that the DSSCs based on XW40/CDCA + Z1 are photostable.

\section{Conclusions}

In summary, a new class of doubly strapped porphyrin sensitizers has been designed and successfully synthesized as DSSC sensitizers. Compared to the dyes with the porphyrin core wrapped with four long alkoxyl chains, the circle chain strapping can further suppress the dye aggregation and charge recombination. Thus, the $V_{\text {oc }}$ was greatly improved from $711 \mathrm{mV}$ (XW10) to $730 \mathrm{mV}$ (XW40). Moreover, the much more compact structures of the strapped porphyrins are favorable for improving the dye loading amount, leading to the improvement of $J_{\mathrm{sc}}$. With the simultaneously improved $V_{\mathrm{oc}}$ and $J_{\mathrm{sc}}$, the efficiency of $9.3 \%$ achieved for the strapped porphyrin was found to be significantly higher than the $8.6 \%$ obtained for the wrapped dye XW10. The auxiliary electron-withdrawing unit BTD in XW41 successfully extended the response spectral range with a striking onset wavelength of $830 \mathrm{~nm}$ in the IPCE spectra, but the extended $\pi$-conjugation framework resulted in a lowered efficiency of $8.2 \%$ because of the aggravated dye aggregation. After applying a combined approach of coadsorption with CDCA and cosensitization with Z1, high efficiencies of $10.6 \%$ and $10.2 \%$ were achieved for XW40 and XW41, respectively. These results provide a novel strategy for developing efficient DSSCs by employing strapped porphyrin dyes.

\section{Conflicts of interest}

There are no conflicts to declare.

\section{Acknowledgements}

This work was financially supported by the Shanghai Municipal Science and Technology Major Project (Grant No. 2018SHZDZX03) and the international cooperation program of the Shanghai Science and Technology Committee (17520750100), NSFC (21472047, 21772041, 201702062, 21811530005, 21878078), Program for Professor of Special Appointment (Eastern Scholar, GZ2016006) at Shanghai Institutions of Higher Learning, Shanghai Pujiang Program (17PJ1401700), Fundamental Research Funds for the Central Universities (WK1616004, 222201717003), and Program of
Introducing Talents of Discipline to Universities (B16017). The authors thank the Research Center of Analysis and Test of the East China University of Science and Technology for the help with the characterization. W. T. is grateful to the China Scholarship Council and British Council for the visiting fellowship.

\section{Notes and references}

1 C. C. Chou, F. C. Hu, H. H. Yeh, H. P. Wu, Y. Chi, J. N. Clifford, E. Palomares, S. H. Liu, P. T. Chou and G. H. Lee, Angew. Chem., Int. Ed., 2014, 53, 178-183.

2 B. O'Regan and M. Grätzel, Nature, 1991, 353, 737-740.

3 C. Li, J. Zhang, J. Song, Y. Xie and J. Jiang, Sci. China: Chem., 2018, 61, 511-514.

4 A. S. Hart, B. K. Chandra, H. B. Gobeze, L. R. Sequeira and F. D'Souza, ACS Appl. Mater. Interfaces, 2013, 5, 5314-5323.

5 T. Higashino, Y. Kurumisawa, N. Cai, Y. Fujimori, Y. Tsuji, S. Nimura, D. M. Packwood, J. Park and H. Imahori, ChemSusChem, 2017, 10, 3347-3351.

6 T. Higashino and H. Imahori, Dalton Trans., 2015, 44, 448463.

7 L. L. Li and E. W. Diau, Chem. Soc. Rev., 2013, 42, 291-304. 8 J. Luo, M. Xu, R. Li, K. W. Huang, C. Jiang, Q. Qi, W. Zeng, J. Zhang, C. Chi, P. Wang and J. Wu, J. Am. Chem. Soc., 2014, 136, 265-272.

9 C. L. Wang, M. Zhang, Y. H. Hsiao, C. K. Tseng, C. L. Liu, M. Xu, P. Wang and C. Y. Lin, Energy Environ. Sci., 2016, 9, 200-206.

10 J. M. Ball, N. K. S. Davis, J. D. Wilkinson, J. Kirkpatrick, J. Teuscher, R. Gunning, H. L. Anderson and H. J. Snaith, RSC Adv., 2012, 2, 6846-6853.

11 C. L. Mai, W. K. Huang, H. P. Lu, C. W. Lee, C. L. Chiu, Y. R. Liang, E. W. Diau and C. Y. Yeh, Chem. Commun., 2010, 46, 809-811.

12 J. W. Shiu, Y. C. Chang, C. Y. Chan, H. P. Wu, H. Y. Hsu, C. L. Wang, C. Y. Lin and E. W. G. Diau, J. Mater. Chem. A, 2015, 3, 1417-1420.

13 F. M. Jradi, D. O'Neil, X. Kang, J. Wong, P. Szymanski, T. C. Parker, H. L. Anderson, M. A. El-Sayed and S. R. Marder, Chem. Mater., 2015, 27, 6305-6313.

14 C. Y. Lin, Y. C. Wang, S. J. Hsu, C. F. Lo and E. W. G. Diau, J. Phys. Chem. C, 2010, 114, 687-693.

15 Enzymes in Industry: Production and Applications, ed. W. Aehle, 3rd edn, Wiley-VCH, Weinheim, 2007.

16 C. L. Wang, C. M. Lan, S. H. Hong, Y. F. Wang, T. Y. Pan, C. W. Chang, H. H. Kuo, M. Y. Kuo, E. W. G. Diau and C. Y. Lin, Energy Environ. Sci., 2012, 5, 6933-6940.

17 Q. Chai, W. Li, Y. Wu, K. Pei, J. Liu, Z. Geng, H. Tian and W. Zhu, ACS Appl. Mater. Interfaces, 2014, 6, 1462114630.

18 A. Yella, C. L. Mai, S. M. Zakeeruddin, S. N. Chang, C. H. Hsieh, C. Y. Yeh and M. Gratzel, Angew. Chem., Int. Ed., 2014, 53, 2973-2977.

19 Y. K. Eom, S. H. Kang, I. T. Choi, Y. Yoo, J. Kim and H. K. Kim, J. Mater. Chem. A, 2017, 5, 2297-2308.

20 H. Song, X. Li, H. Ågren and Y. Xie, Dyes Pigm., 2017, 137, 421-429. 
21 P. Brogdon, H. Cheema and J. H. Delcamp, ChemSusChem, 2018, 11, 86-103.

22 H. Li, M. Fang, R. Tang, Y. Hou, Q. Liao, A. Mei, H. Han, Q. Li and Z. Li, J. Mater. Chem. A, 2016, 4, 16403-16409.

23 Y. Ren, D. Sun, Y. Cao, H. N. Tsao, Y. Yuan, S. M. Zakeeruddin, P. Wang and M. Gratzel, J. Am. Chem. Soc., 2018, 140, 2405-2408.

24 Y. Liu, Y. Cao, W. Zhang, M. Stojanovic, M. I. Dar, P. Péchy, Y. Saygili, A. Hagfeldt, S. M. Zakeeruddin and M. Grätzel, Angew. Chem., Int. Ed., 2018, 57, 14125-14128.

25 J. Liu, Y. Numata, C. Qin, A. Islam, X. Yang and L. Han, Chem. Commun., 2013, 49, 7587-7589.

26 J. Liu, X. Yang, A. Islam, Y. Numata, S. Zhang, N. T. Salim, H. Chen and L. Han, J. Mater. Chem. A, 2013, 1, 10889-10897.

27 M. Ikai, F. Ishikawa, N. Aratani, A. Osuka, S. Kawabata, T. Kajioka, H. Takeuchi, H. Fujikawa and Y. Taga, Adv. Funct. Mater., 2006, 16, 515-519.

28 V. Rauch, J. Conradt, M. Takahashi, M. Kanesato, J. A. Wytko, Y. Kikkawa, H. Kalt and J. Weiss, J. Porphyrins Phthalocyanines, 2013, 18, 67-75.

29 C. H. Lee and E. Galoppini, J. Org. Chem., 2010, 75, 36923704.

30 S. Fan, K. Lv, H. Sun, G. Zhou and Z. S. Wang, J. Power Sources, 2015, 279, 36-47.
31 M. W. Lee, J. Y. Kim, H. J. Son, J. Y. Kim, B. Kim, H. Kim, D. K. Lee, K. Kim, D. H. Lee and M. J. Ko, Sci. Rep., 2015, 5, 7711.

32 Y. Gao, X. Li, Y. Hu, Y. Fan, J. Yuan, N. Robertson, J. Hua and S. R. Marder, J. Mater. Chem. A, 2016, 4, 12865-12877.

33 Y. Xie, Y. Tang, W. Wu, Y. Wang, J. Liu, X. Li, H. Tian and W. H. Zhu, J. Am. Chem. Soc., 2015, 137, 14055-14058.

34 Q. Xu, G. Yang, Y. Ren, F. Lu, N. Zhang, M. Qamar, M. Yang, B. Zhang and Y. Feng, Phys. Chem. Chem. Phys., 2017, 19, 28867-28875.

35 X. Li, X. Zhang, J. Hua and H. Tian, Mol. Syst. Des. Eng., 2017, 2, 98-122.

36 J. Luo, X. Wang, L. Fan, G. Li, Q. Qi, K. W. Huang, T. L. Dexter Tam, J. Zhang, Q. Wang and J. Wu, J. Mater. Chem. C, 2016, 4, 3709-3714.

37 Y. Cui, Y. Wu, X. Lu, X. Zhang, G. Zhou, F. B. Miapeh, W. Zhu and Z. S. Wang, Chem. Mater., 2011, 23, 4394-4401.

38 Y. Tang, Y. Wang, X. Li, H. Ågren, W. H. Zhu and Y. Xie, ACS Appl. Mater. Interfaces, 2015, 7, 27976-27985.

39 J. Liu, B. Liu, Y. Tang, W. Zhang, W. Wu, Y. Xie and W. H. Zhu, J. Mater. Chem. C, 2015, 3, 11144-11150.

40 Y. Wang, B. Chen, W. Wu, X. Li, W. Zhu, H. Tian and Y. Xie, Angew. Chem., Int. Ed., 2014, 53, 10779-10783. 